\title{
CAGP-CCSMH Annual Scientific Meeting \\ Canadian Academy of Geriatric Psychiatry and Canadian \\ Coalition for Seniors' Mental Health
}

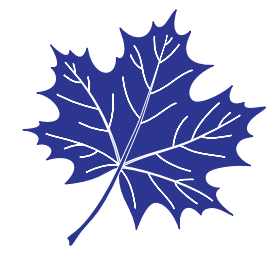

https://doi.org

\author{
Supporting Seniors' Mental Health Through Age-Friendly \\ Community Approaches in Atlantic Canada
}

Elaine Jones-McLean

The Canadian population is aging and the Atlantic Provinces have the oldest population in the country. Mental health is an integral part of healthy aging, and multi-sectoral approaches to promoting the mental health of older adults are important. The Age-Friendly Communities (AFCs) approach, a global initiative launched by the WHO in 2006, supports active aging in communities by designing policies, programs, services and the built and social environments in a way that facilitates safe, engaged, and healthy aging. Each of the eight domains included in the AFC approach has an impact on mental health. These include: outdoor spaces and buildings; transportation; housing; social participation; respect and social inclusion; civic participation and employment; communication and information; and community support and health services. The AFC approach addresses seniors' mental health across the prevention and treatment spectrum, from building social connectedness to preventing loneliness, through to linking with specialized geriatric and/or psychiatry clinical care. This symposium will share initiatives and perspectives from Atlantic Canada that promote healthy active aging including positive mental health from a multidisciplinary perspective that includes the community environment and clinical care.

Age-Friendly Communities: Using Indicators to Understand Age Friendliness as it Relates to Mental Health in Atlantic Canada

\section{Heather Orpana}

Background: Age Friendly Communities support active, healthy aging through promoting supportive built and social environments, as well as programs, policies and health and community services that are oriented towards the needs of older adults. This presentation will describe the process undertaken by the Public Health Agency of Canada to develop a set of indicators across the eight AFC domains, and how these indicators relate to the mental health of older adults with a focus on Atlantic Canada.

Methods: An iterative, consultative process was conducted to develop a set of indicators for evaluation for AFCs. A literature review and environmental scan identified a number of potential indicators. Expert and stakeholder consultations were conducted to rate indicators based on importance, actionability and feasibility. The final list of proposed indicators was chosen based on these results as well as policy and alignment considerations.

Results: Thirty-nine indicators were identified across the eight AFC domains, as well as four indicators of health and social outcomes. These indicators can be used to understand aspects of the age friendliness of communities and how age friendliness relates to the mental health of seniors. Examples using data from Atlantic Canada as a whole will be presented.

Conclusions: The indicators can be used by communities to gauge their progress on the road to becoming more agefriendly, in a way that is tailored to specific settings and times. Examples of indicators and supporting data sources in Atlantic Canada, as well as what insights such data can provide to inform decision making around actions to support seniors' mental health will be discussed.

Social Vulnerability and Connections in the Clinical Care of Seniors

\section{Melissa K. Andrew}

Background: Social circumstances are important contributors to older adults' health. In clinical care, social factors are important in a bidirectional way, both as risk factors (or supportive enablers) for health outcomes, and as pragmatic considerations when care and supports are being planned and evaluated. Given that each individual's social circumstances are uniquely complex, a unified view of social circumstances is useful. With this in mind, the concept of social vulnerability is discussed. 
Social vulnerability stems from the accumulation of multiple and varied social problems. Importantly, the social factors that contribute to overall social vulnerability come into play at different levels of influence (individual, family and friends, peer groups, institutions and society at large). A social ecology perspective is discussed as a useful framework for considering social vulnerability, as it allows for attention to each of these levels of influence.

In this presentation, the relevance of clinical and epidemiological understanding of social vulnerability will be discussed in relation to age friendly cities initiatives.

Methods: To be discussed.

Results: To be discussed.

Conclusions: To be discussed.

\section{Age-Friendly Initiatives in Summerside PEI - Building a Community Legacy of Seniors' Inclusion}

Judy-Lynn Richards

Background: Recent research provides links among the built environments and seniors' mental health, where communities can build social capital, promote belongingness, a positive outlook on aging, and reinforce seniors' contributions. Like its Maritime neighbours, PEI has a high proportion (18.9\%) and growing number of seniors (PEI Government, 2017). Summerside, PEI is one of Canada's AFC Initiatives, in its fifth of a five-year WHO AFC process; its current focus is on developing its legacy plan to share with communities across PEI. Summerside has key lessons, best practices, and challenges to share about fostering AFC initiatives in the Maritimes and across Canada.

Methods: This presentation will describe the planning/ implementation of Summerside's AFC initiative, its path, current status and the City's next steps and directions for this initiative. Several successes will be highlighted including initiatives that address seniors' well-being and social isolation.

Results: Consistent with evidence-based research that link positive mental health to the social and physical built environment, social connectivity and empowerment, Summerside's AFC Committee has developed many smaller/ larger projects to develop a City embracing aging/seniors. The projects include many initiatives from a City-wide assessment of its age-friendliness, training/certifying businesses as age-friendly, facilitating intergenerational relations, promoting partnerships that enhance seniors' community contributions, an age-friendly housing project, to a commemorative quilt.
Conclusions: Simple to complex age-friendly projects, any of which will contribute to seniors' wellness, are an important aspect of seniors' mental health. This session will provide examples and insights for other communities looking to embark on similar initiatives for this segment of the population.

\section{Collaborative Governance to Meet the Challenge of Aging: The Case of Age-Friendly Cities}

Mario Paris

Background: The goal of my presentation is to explain the importance of collaborative governance within Age-Friendly Cities (AFC).

Methods: I use multiple case studies of four very different municipalities within the AFC financed by the Canadian Institutes of Health Research. In this presentation, I look at collaborative governance through four conceptual dimensions (Ansell and Gash, 2008).

Results: Starting conditions: Our results show that inequalities in resources, as well as in power relationships, exist between different actors on the steering committee, but also within the same group of actors. In addition, the AFC process offers the conditions to incite actors to engage in improving the quality of life of older adults, but the engagement is not guaranteed if the starting conditions change. Our results show that the relationship among the members of the steering committee is based on openness and sociability.

Facilitative leadership: If the leadership can be expressed by a hierarchical position of an actor upon the other actors, another kind of leadership is also present. This one is built on respect and trust that one actor inspires others by her or his efficiency.

Institutional design: The institutionalization of the guidelines of the AFC processes can increase the degree of municipal bureaucratization and of new formal and informal procedures created to improve the functioning of collaborative governance.

Collaborative process: The AFC provides a clear and structured process of collaborative governance. I observed five variables around this process: 1) face-to-face dialogue, 2) trust building, 3) commitment to the process, 4) share understanding, and 5) the intermediate outcomes.

Conclusions: From numerous examples and explanations, the Ansell and Gash (2008) model constitute a conceptual framework appropriate to understand the complexity of the Age-Friendly Cities. 
Collaborative Care for Psychiatric Disorders in Older Adults: From Evidence to Practice

Nick Kates, Tarek Rajji, Karen Saperson, Fiona Parascandalo

Background: The prevalence of mild cognitive impairment, depression and anxiety in individuals over the age of 60 being seen in primary care is high, but these problems frequently go unrecognized and untreated although there is increasing evidence that evidence based interventions in primary care can increase identification and treatment rates for individuals with depression, anxiety and mild cognitive impairment.

Methods: This symposium describes a project with two goals. The first was to design and introduce an evidence-based pathway for the recognition and treatment of $\mathrm{MCI}$ and accompanying depression and anxiety within the offices of over 20 family physicians in two cities. The second was to identify changes and system adjustments practices needed to make to accommodate the new program and the pathway. To do this, 2 cohorts of patients were identified in each physician's patient as the study group - everyone born in 1951 and everyone born in 1956, with the populations being born in 1950 and 1955 being used as controls.

Results: The symposium summarises the pathway developed, the process for introducing into primary care, the adjustments that primary care practices needed to make to accommodate a change in their routines, and the results of focus groups held with primary care practices six months after starting the project. It describes the characteristics of the populations being seen and presents initial data from the first 12 months of the study, the problems identified, and the challenges the project has faced and overcome to date.

Conclusions: It is early in the project, but there is clear evidence of the appetite for primary care providers for assistance with managing seniors with cognitive and mood-related problems, of the need to tailor or adapt existing guidelines to fit with the realities of primary care and of the willingness of primary care practices to make adjustments to accommodate the new protocol.

Inter-Disciplinary Approach to Design and Implementation of a CBT group for Depression in Older Adults (CBT-GD)

Donna Pfefer Litman, Petal Abdool, Karen Ward, Holly Ito, Camilo Yang

Background: Depression is the most common mental health problem in older adults. As the geriatric population grows, the demand on the mental health system will escalate. Cognitive-
Behavioural Therapy (CBT) is one of the most effective psychotherapeutic interventions for treatment of depression. Challenges and needs of older adults differ from the general population. Due to the high demand, group format allows for more timely treatment provision.

Methods: An interdisciplinary team developed a manual to meet the needs of this population. The Geriatric Depression Scale (GDS) was administered pre and post-group, the Quick Inventory of Depression Self Report (QIDS-SR) was completed weekly. Participants were $60+$ with a diagnosis of major depressive disorder. Exclusion criteria included diagnoses that would pose a barrier to learning such as Neurocognitive Disorders.

Results: To date five CBT-GD groups were completed. Eighty-two individuals were screened for suitability. A total of 35 participants were enrolled with an attrition rate of $14 \%$. The total mean QIDS-SR score pre-group was $12.4(\mathrm{SD}=$ $5.5, \mathrm{~N}=28)$ and post-group was $8.6(\mathrm{SD}=5.1 ; \mathrm{N}=28)$. The total mean GDS score pre-group was $9.2(\mathrm{SD}=2.9, \mathrm{~N}=21)$ and post-group was of $6.9(\mathrm{SD}=3.4 ; \mathrm{N}=21)$.

Conclusions: Depressive symptoms improved in those who completed the 12 week CBT-GD group. This format allows for timely, efficient access to CBT. Barriers encountered were co-morbid diagnoses, relatively small number of participants to date, and need for improved data collection. Future considerations include further refining of the CBT-GD manual through improved capture of qualitative feedback from participants.

\section{Diagnosing Major Neurocognitive Disorders in Individuals with Intellectual Disability}

Catherine Chenge, Ade Orimalade

Background: As individuals with intellectual disability (ID) age, the risk of developing age-related conditions, such as a major neurocognitive disorder increases. Though an association has been established between older adults with Down Syndrome (DS) and an increased risk for Alzheimer's disease (AD), less is known about older adults with other IDs. The evaluation and diagnosis of a major neurocognitive disorder in older adults with ID can be challenging and requires a modified approach. Dementia may be more common in older adults with intellectual disability than in the general geriatric population.

Methods: A literature review was conducted regarding major neurocognitive disorders and intellectual disability, with a focus on screening and diagnosis.

Results: The primary objectives were to determine the prevalence of dementia in older adults with ID, and to review 
current knowledge and guidelines for the diagnoses of dementia in this population. The clinical approach to evaluation, including unique considerations and the use of screening tools is discussed. The incidence of dementia in older people with intellectual disabilities is up to five times higher than older adults in the general population. Studies also suggest that by age 40 , all adults with DS demonstrate some degree of neuropathologic defects postmortem that meet criteria for AD.

Conclusions: Early recognition and proper monitoring of elderly individuals with ID experiencing cognitive and/or functional decline is essential to improving health outcomes, meeting care needs and improving quality of life in this population. More research is needed, as current literature on dementia and intellectual disability in the elderly remains limited.

\section{Dementia: Ambiguous Loss and Grief}

Mary Schulz

Background: Family members of people with dementia often experience a continuous and profound sense of loss and subsequent grief as they live through the progression of the disease. "Dementia creates ambiguous loss. The duality of your loved one's being absent and present at the same time is confusing, and finding meaning (or making sense of your situation) becomes immensely challenging. Without meaning, it's hard to cope". (P.Boss, 2011) Unfortunately, there is a dearth of educational resources and support groups on ambiguous loss and grief related to dementia.

Methods: The Alzheimer Society of Canada (ASC) conducted thorough research reviews and Pan-Canadian interviews with health-care providers, individuals with dementia and caregivers who have benefited from grief and loss interventions in order to better understand this issue and to inform the development of educational resources.

Results: ASC developed two resources to identify, acknowledge and normalize the feelings of loss and grief experienced by caregivers of people with dementia - from diagnosis to end-of-life and after caregiving- while providing strategies for understanding and responding to grief reactions. The first resource targets healthcare providers in order to help them understand this phenomena and the second is designed for people with dementia, families and their support network.

Conclusions: The goal of this session is to address grief, validate the experience of ambiguous loss and equip family and professional caregivers with practical strategies to help them support people with dementia from the initial onset of symptoms and diagnosis to end-of-life and life after caregiving.
Facts, Feelings and Fears: Supporting Families Through Dementia's End-of-Life

Mary Schulz

Background: As of 2016, 564,000 Canadians are living with Alzheimer's disease and other dementias (Alzheimer Society of Canada, 2016). Many diseases can cause dementia, the most common being Alzheimer's disease. Alzheimer's disease is a fatal disease that eventually affects all aspects of a person's life: how they think, feel, and act. People with dementia can therefore benefit from a palliative approach, long before death is near. However, palliative care has typically not been available to people whose prognosis is uncertain and where the dying process is protracted (Small, 2007).

Methods: The Alzheimer Society of Canada has developed an online resource to help families prepare for end-of-life, make some of the difficult decisions they may face, and cope with the grief they may experience. The rationale for this resource, strategies for use and how to access it will be presented, along with resources available to support health care providers in their work in end-of-life dementia care.

Results: Resources are available to assist health care providers in supporting a person centered approach to palliative end-of-life dementia care as well as strategies that can lead to a more comfortable end-of-life experience for the person with dementia and their families.

Conclusions: While it is human nature to avoid talking about sad and difficult topics such as end-of-life, people impacted by dementia are hungry for practical, reliable information about what to expect at this stage. Health care providers have an important role to play in preparing people with dementia and families for end-of-life.

Considering a Career in Geriatric Psychiatry? Meet the Experts in Education, Research, Advocacy and Community Practice

Paul Blackburn, Beverly Cassidy, David Conn, A. Baillod, V. Thoo, C. Lazaro, Amanda Canfield, E. Gregory, D. Bhangoo, S. Chaudhari, O. Yashchuk, A. Myles

Background: The Canadian Academy of Geriatric Psychiatry (CAGP) Trainee Strategy formed in 2011 with the goal of creating opportunities and support for Member-in-Training (MIT) CAGP Members.

Formal and informal feedback has shown that trainees often wonder "What is a geriatric psychiatrist?" and "What would a career in geriatric psychiatry entail?" Trainees have indicated that they are interested in learning more about the lifestyle and career of a geriatric psychiatrist from experts in the field. 
In response to trainees' feedback, an interactive workshop has been created by the CAGP Trainee Strategy to explore career opportunities within geriatric psychiatry. This workshop will involve a brief introduction of the topic followed by a 30-minute panel discussion by experts in the field of geriatric psychiatry. Additionally, it is the intention of the workshop authors to administer a brief pre-ASM needs assessment questionnaire to MIT and affiliate in training (AIT) members who will be attending the ASM so as to determine specific questions and areas of interest.

The expert panel will be asked to describe why they chose a career in geriatric psychiatry while highlighting the various opportunities they have had during their careers and share their perspective of the lifestyle of a geriatric psychiatrist. They will also be asked to respond to questions arising from the brief needs assessment.

The second half of the workshop will involve trainees interacting within small groups in focused discussions with the panel experts. This is a great opportunity for residents, fellows, and students from all disciplines to network with experts and colleagues, and ask any questions they have about their career development in a friendly setting.

Psychotic, Rigid, and Febrile: Deciding to Stop Antipsychotic Medication in a Patient with Both Neuroleptic Malignant Syndrome and Severe and Persistent Mental Illness

\section{Sarah Levitt, Robert Madan}

Background: A 69 year-old woman with Bipolar Disorder (BD) was admitted to a rehabilitation unit after an elective right knee replacement. This patient's history included lengthy psychiatric hospitalizations, multiple suicide attempts, and BD which had proven refractory to many medication regimens. In the past decade, our patient had achieved a period of stability with concurrent use of risperidone and quetiapine. Her longstanding antipsychotic medication regimen was abruptly stopped in the post-operative period and restarted upon admission to the rehabilitation unit. Five days after restarting antipsychotics this patient became intermittently febrile with hypersomnolence, psychomotor retardation, and rigidity.

Methods: Using an interactive discussion format, the case will be reviewed in terms of the differential diagnosis and evidence-based care when Neuroleptic Malignant Syndrome (NMS) presents in a medically and psychiatrically complex patient. The discussion will be supplemented by a review of the scientific literature on diagnostic and treatment considerations for NMS, highlighting the considerations when managing a geriatric patient with severe and persistent mental illness (SPMI). The impact of discontinuing antipsychotics secondary to NMS in an individual with BD will be discussed as it pertains to risk-benefit discussions in planning immediate and long-term treatment with patients and their families.

Results: This patient was treated with cessation of all antipsychotics. The life-threatening ramifications of NMS were discussed with the patient and her family, as well as the potential impact of discontinuing the antipsychotics on the course of her affective illness. Geriatric medicine assisted in the management of medical comorbidities in the context of NMS. Eventual transfer to an acute care hospital was necessary.

Conclusions: NMS poses unique treatment considerations in older adults with SPMI. Clear communication is required with patients and their families in order to assist them in making informed decisions.

\section{Mobilizing the Uptake of the Guidelines for Comprehensive Mental Health Services for Older Adults in Canada}

Francine Knoops, Marie-France Rivard, Sareda Quah-Vo, Kimberley Wilson, Simone Powell

Background: In 2011 the Mental Health Commission of Canada (MHCC) published Canadian Guidelines for Comprehensive Mental Health Services for Older Adults in Canada. They offer a blueprint for action to promote mental health and integrate services to meet the complex mental health needs of Canada's aging population. In 2017, the MHCC initiated knowledge exchange (KE) to promote the Guidelines and policy development on home care to apply its principles.

Methods: A scan of the presence of the Guidelines in academic literature was conducted, along with a survey and key informant telephone interviews to collect practice-based evidence about their impact. A scoping review of the literature and analysis of policy documents were undertaken to assess attention to mental health in home care. Two roundtables identified barriers, priority issues and opportunities for action.

Results: Findings confirmed limited uptake of the Guidelines and limited focus on mental health in home care in Canada. Stakeholders identified four priorities for KE: a) get seniors mental health on policy agendas; b) bring out diverse voices of seniors' lived experience; c) make the guidelines more accessible; and, d) professional education to address interprofessional collaboration and ageism. Eight policy areas in home care were identified: training, foundational principles, technology and access, integration and support for collaboration, 
diverse populations, service eligibility and flexibility, family and person-centred care, lived experience as expertise.

Conclusions: Implementing the Guidelines involves many stakeholders and a long-term view. Current MHCC activities offer first steps to support this work of many moving forward.

\section{The Use of ECT in Patients with BPSD}

Sameh Hassan, Mike Flynn, Shabbir Amanullah, K.S. Shivakumar, P. Krishna

Background: Behavioral and psychological symptoms in Dementia (BPSD) occur in the majority of patients with advancing Major Neurocognitive Disorder (Dementia). Many of these patients with BPSD show agitation and aggression. In some of these severe cases environmental, behavioural and pharmacological interventions are not sufficient to manage these disturbing and sometimes dangerous behaviours. Electroconvulsive therapy (ECT) can be a viable and safe intervention in managing these problematic behaviours; both as a short and long-term intervention.

Methods: PubMed search has shown relevant articles available till March 2018 is done using key words "ECT, agitation, dementia"

Results: The published systematic review yielded 264 articles (Van den berg, Am J Psych 2017). Only one study was a prospective cohort study and many are case reports or case series. There are no prospective randomized studies to date. Out of 122 patients, clinically significant improvement is observed in $88 \%$ of the patients.

Conclusions: ECT could be an effective treatment for severe treatment refractory agitation and aggression in patients with major neurocognitive disorders. This can reduce care giver burden, and improve safety and quality of life for patients and care providers in long-term care settings. Cognitive effects are transient even in patients with cognitive impairment. Further prospective randomized studies are needed to further substantiate these results.

\section{Development and Evaluation of Web Application (DObs) for Direct Behavioural Observation in Dementia}

Andrea Iaboni, Cecelia Marshall, Mario Tsokas, Lori Schindel-Martin

Background: A principle of assessment of behavioural symptoms in dementia is to chart observed behaviours at regular intervals, to establish their frequency, severity, and any patterns. We have developed a web-based mobile Dementia Observation (DObs) application designed for use by front-line staff, with the goal of improving the ease, completeness, and accuracy of behavioral data collection. We will present the development and evaluation of this application.

Methods: We tested the application in usability assessments and in clinical use. Participants were nursing staff on the Toronto Rehab inpatient dementia unit. Participants completed scales and qualitative data was collected via post-test questions.

Results: We conducted several iterations of usability testing and app improvement. In the clinical usability testing, participants completed $100 \%$ of the initiation tasks correctly, but only $66 \%$ of the observation tasks and $40 \%$ of the completion tasks. On average, participants entered observation data 1.5 times per interval, responded to notifications within $5 \mathrm{sec}-$ onds, and took 30 seconds to enter an observation. Many of the failed tasks were related to challenges in incorporating the mobile device into the clinical workflow and technical failures related to an unfamiliar mobile device.

Conclusions: Mobile technology offers an opportunity to improve the assessment and treatment of responsive behaviours in dementia. Two broad areas of improvement were identified: first, with respect to the usability of the DObs mobile application itself, and second, with respect to the adaptability and comfort of staff in using a mobile device for clinical data collection at the bedside.

\section{Clinical Guidelines on Substance Use Disorders Among Older Canadians: Development and Key Recommendations}

David Conn, Jonathan Bertram, Peter Butt, David Hogan, Meldon Kahan, Amy Porath, Launette Rieb, Marilyn WhiteCampbell, Dallas Seitz, Kiran Rabheru

Background: Older adults face unique physiological, psychological, social and pharmacological circumstances that make them more vulnerable to the effects of substances. While still woefully under-studied, there is mounting evidence of the prevalence of Alcohol, Benzodiazepine, Cannabis and Opioid Use Disorders among older adults, including Canadians.

Methods: Health Canada has funded the Canadian Coalition for Seniors' Mental Health (CCSMH) to develop national clinical guidelines for substance use disorders among older adults. By convening working groups with members from across Canada and from diverse disciplines (including geriatric psychiatry, addictions medicine, pharmacy, family medicine, psychology, social work, nursing, policy and people with 
lived experience), CCSMH developed a process to address the unique challenges of developing clinical guidelines focused on the prevention, screening, assessment and treatment of Alcohol, Benzodiazepine, Cannabis and Opioid Use Disorders among older adults.

Results: While the Clinical Guidelines will be formally released in November 2018, key highlights include Low Risk Drinking Guidelines for older adults, recommendations regarding the treatment of opioid use disorder in the context of chronic pain management among older adults, calls for an overall reduction in the prescription of benzodiazepines, and descriptions of clinicians' role in assessing cannabis use in the elderly in light of new legislation.

Conclusions: Reported rates of substance use disorder among older Canadians demonstrate the need for clear, pragmatic, evidence-based clinical guidelines for the prevention, screening, assessment and treatment of Alcohol, Benzodiazepine, Cannabis and Opioid Use Disorders that are specifically designed for older Canadians.

\section{Substance Use Disorders Among Older Adults}

David Gardner, Karen Reimers, Nancy Vasil

Background: Clinicians and community-based service providers are increasingly encountering problematic substance use and Substance Use Disorder among older adults in their daily work. Problematic substance use among older adults often occurs unintentionally and insidiously through overprescription of substances, inappropriate polypharmacy, and recreational use. Among older adults, substance use disorders are often associated with concurrent mental illnesses, cognitive disorders, physical illness/frailty, and psychosocial stresses often experienced by older adults such as social isolation, retirement, and bereavement.

Methods: The area of substance use disorders in older adults is understudied and underreported in the psychiatry, addictions, and gerontology literature. This research gap presents challenges to front line workers.

Results: While still woefully under-studied, there is mounting evidence of the prevalence of Alcohol, Benzodiazepine, Cannabis and Opioid Use Disorders among older adults, including Canadians. Older adults face unique physiological, psychological, social and pharmacological circumstances that make them more vulnerable to the effects of substances.

Conclusions: Substance use disorder among older adults is often unrecognized and untreated. In order to deal effectively with this "invisible epidemic", clear, pragmatic, evidencebased clinical guidelines are needed for the prevention, screening, assessment and treatment of Alcohol, Benzodiazepine, Cannabis and Opioid Use Disorders that are specifically designed for older Canadians.

\section{Development of National Clinical Guidelines on Substance Use Disorders Among Older Canadians}

David Conn, Keri-Leigh Cassidy, Lori Amdam, Ashok Krishnamoorthy

Background: While several clinical guidelines address substance use disorder (SUD), they rarely target older adults. Developing national clinical guidelines requires intense, challenging and collaborative, multi-disciplinary work.

Methods: By convening working groups (WGs) with members from across Canada and from diverse disciplines (including geriatric psychiatry, addictions medicine, pharmacy, family medicine, psychology, social work, nursing, policy and people with lived experience), the Canadian Coalition for Seniors' Mental Health (CCSMH) embarked on the process of developing clinical guidelines focused on SUDs among older adults.

Results: WGs used shared online databases to collaborate and met through a combination of virtual platforms and in-person working meetings. The CCSMH facilitated cross-pollination of expertise across WGs, called on international experts as needed, and created platforms through which challenges, strategies and solutions could be shared across WGs. This included having the co-chairs of each WG participate in an overarching Steering Committee and ensuring continuity through consistent staff support across WGs. At a 2-day national meeting, all 4 WGs convened to share updates, successes and struggles. Each of the WGs created subgroups to develop the subsections of prevention, screening, assessment, treatment of SUDs, and a special populations section. Preliminary recommendations were reviewed by the whole WG. Where necessary and appropriate, data from younger or mixed-age samples was extrapolated. The GRADE tool was used to assess quality of evidence and rate strength of recommendations.

Conclusions: By convening multi-disciplinary teams, using available technology, facilitating cross-pollination, sharing of expertise and communicating openly about challenges and potential solutions, CCSMH was able to address key challenges inherent in the development of clinical guidelines on SUD among older adults. 


\section{Key Recommendations from Clinical Guidelines on Substance Use Disorders Among Older Canadians}

\author{
Kiran Rabheru, Christopher Kitamura, Dallas Seitz, Lisa \\ Van Bussel
}

Background: Health Canada has funded the Canadian Coalition for Seniors' Mental Health (CCSMH) to develop national clinical guidelines for substance use disorders among older adults.

Methods: CCSMH convened working groups with members from across Canada and from diverse disciplines (including geriatric psychiatry, addictions medicine, pharmacy, family medicine, psychology, social work, nursing, policy and people with lived experience) to develop clinical guidelines focused on the prevention, screening, assessment and treatment of Alcohol, Benzodiazepine, Cannabis and Opioid Use Disorders among older adults.

Results: While the clinical guidelines will be formally released in November 2018, key highlights include Low Risk Drinking Guidelines for older adults, recommendations regarding the treatment of opioid use disorder in the context of chronic pain management among older adults, calls for an overall reduction in the prescription of benzodiazepines, and descriptions of the clinician's role in assessing cannabis use in the elderly in light of new legislation.

Conclusions: In addition to developing clinical guidelines for the prevention, screening, assessment and treatment of Alcohol, Benzodiazepine, Cannabis and Opioid Use Disorders that are specifically designed for older Canadians, CCSMH will ensure broad dissemination and uptake of the guidelines through extensive knowledge translation (KT) activities. This includes the development of KT tools such as clinical pocket cards, presentations and webinars for healthcare professionals, as well as brochures, info-sheets, webinars and presentations designed for older adults, their families and caregivers. We will also explore ways to harness the rapidly expanding area of social media by clinicians and consumers alike.

\section{Meaning, Purpose and Personhood in Long-Term Care Settings}

Shabbir Amanullah, Sean Welbourn, Mark Dager, Karen Cassells, Amy Swayze, Randi Williams

Background: Aging research and aging theories, well studied since the 1950's examine the global definition of aging as an accumulation of inevitable changes that occur over the span of a lifetime. "No single theory is generally accepted.
Theories that can contribute to the important practical goal of increasing the healthy, useful span of humans will endure" (Ann N Y Acad Sci. 1998 Nov 20; 854:1-7. Aging: phenomena and theories). Aging however is not merely the passage of time. "It is the manifestation of biological events that occur over a span of time. Often the progression of change is subtle. There is no perfect definition of aging but, as with love and beauty, most of us know it when we experience it or see it" (Hayflick, 1994).

Since the beginning of time scientific findings have confirmed "all things age" and human ageing is universal, irreversible and involves change. Individuals of all age hold age related apprehensions and concerns regarding this unavoidable stage of development and "the formation of each individual's perceptions and experiences of ageing is a dynamic process that pertains to self, social norms and their interplay (Steverink et al., 2001), reflecting the way an individual internalizes social norms" (Levy, 2003; Westerhof and Barrett, 2005). Most often an individual will view themselves as being younger than they actually are. Industry and society reinforce this belief, generating billions in the quest for the proverbial "fountain of youth" and a profound emphasis and glamorization of various anti-aging trends.

Methods: 1. The methodology of enhancing personhood involves collaboration with the individual and shared partnerships as well as identifying the nature surrounding the presenting concerns. (This process involves dialogue between the system i.e hospital staff, LTC staff and community resources and has integrated the person and supports systems into establishing as plan of care prior to admission date to the home.)

2. The goal of decreasing responsive incidents on transition to Long Term care is important to focus on the individual and ease of system changes in environment to help the individual in their illness to adapt these paramount transitions with the goal of decreasing the use of medications sought out early in the process when otherwise the individuals personhood has not been identified.(This process involves a gradual system approach to the admission day by having the pending individual awaiting LTC come to the home prior when within close parameter of time frame to be accepted to the home and participate in the activities of the home with their family member as they would when admitted. This could entail eating meals in communal environment with others to adapt to this scenario, participating in programs within the home, being exposed and introduced to the distinctions that serve LTC including and not exclusive to Nurse Practitioner, OT, PT, Social work, Dietary, Pharmacy, Pastoral care, Recreational Therapist and community linkage to Behavioural Supports Ontario mobile team at a scheduled 1 facility and access to a Geriatric Psychiatrist.)

3.Enhancing the knowledge exchange with all community partners is at the forefront to ensure ease of transition and to develop a universal language and understanding through the identified processes to support all individuals who share the 
common goal of increasing and improving the quality of life of the individuals we serve.(This process was completed by hosting educational events within the community with the title of the workshops being "Sharing the Vision for Excellence in Long Term Care" this event has been offered to all LTCH in Oxford County as well as hospital staff serving and providing education to over 80 individuals per sessions on three separate occasions to date since the initiation of the workshop.)

Results: To date, the results of our comparative scenarios include a retirement home transition to LTC when the client was in crisis need avoiding hospital admission and transition planning was utilized following this model with no hospitalization. This resident previous had a hospitalization while living in retirement setting. With transition dialogue and collaboration this individual was successfully integrated to LTC with combined efforts of all support systems and did not require any further transfer to hospital. This resident thrived in her new environment and adjusted nicely.

The next case involved a resident with multiple occurrences of responsive behaviours when residing in a previous LTC home, this home was unable to support this individuals needs and therefore was required to send to hospital for an extended stay on a secured environment. This processed occupied a needed Mental Health bed, caused concerns to family and the resident as well. IN collaboration between the hospital and receiving LTC facility the care plan needs and supports were reflected to assist in a timely, smooth transition to another LTCH and after 3 years has not resulted in a hospitalization. THe accessibility to Geriatric Psychiatrist and mobile staff with developed care rounds and discussions has ensured weekly to monthly reviews of the resident and supported her changing needs.

IN another case, a resident who residing in hospital was on a complex continuing care unit and hospital length of stay was in excess of 400 days was well transitioned to new LTC setting with the collaboration piece mentioned previously, and has therefore remained in LTC well support with no further needed transitions for stay at hospital.

Another avenue to consider is a community resident coming to respite stay in LTC who had not been separated from spouse in their years of marriage, he had participated in adult day programs in community which provided supports required to spouse to facilitate outings for herself, yet however beneficial and needed these programs are, it did not offer the transitional support to a LTC setting. This resident still awaits LTC placement, in partnership with pending receiving facility and hospital staff the resident and spouse remain supported and needs continually reviewed to make the transition smooth.

Conclusions: Currently this study continues to be piloted and new data and research are pending for additional supports from external partners. To date we can offer positive observation data and willing to showcase more quantitative data for review.
From Disabling Transitions to (Re)Stabilizing Care: Analyzing the Globacl Challenge of Dementia from a Service Delivery Perspective

Katie Aubrecht

Background: Worldwide, there is increasing attention to the impact of dementia on health and social care systems. International health policy responses to the "rising tide" of dementia have been formalized through the establishment of global governance bodies such as the World Dementia Council, and actioned through the development of global surveillance and knowledge sharing platforms such as the World Health Organization (WHO) Global Dementia Observatory, and the Draft Global Action Plan on the Public Response to Dementia.

Methods: A descriptive analysis of policy responses to the global challenge of dementia that focuses on person- and family-centred service delivery.

Results: The evolving and increasing complexity of care associated with dementia often manifests in transitions that people living with dementia make within and across care settings, particularly as dementia progresses. Despite progress in addressing transitions, national dementia strategies have primarily focused on what happens in early transitions and care settings, rather than later transitions and transition points. Integrated care, intersectoral collaboration, and meaningful service user engagement in decision making are three areas where dementia strategies are beginning to be realized in service delivery policy. All three intersect in the introduction of geriatric transitional support and behavioural stabilization units - an emerging best practice approach to complex dementia care, and a mechanism for supporting appropriate transitions across care settings.

Conclusions: The emergence of behavioural stabilization units are discussed as a case that illustrates one way that the global challenge of dementia is being addressed at the local level.

\section{Pharmacological and Clinical Profile of Newer Atypical Antipsychotics as Treatments for Bipolar Disorder: A Systematic Review and Considerations for Use in Older Patients}

Akshya Vasudev, Sumit Chaudari

Background: Bipolar disorder prevalence rates vary in the older adult population (defined as age 65 and above) ranging from $1 \%$ in community dwellers to as high as $8 \%$ to $10 \%$ in 
hospital inpatients. Older agents including lithium and valproic acid offer significant anti-manic efficacy as supported by a recent randomized controlled trial (RCT). However, little is known about their antidepressant mood stabilization potential. Newer atypical antipsychotics are of interest based on their claimed efficacy and tolerability profile. We evaluated the safety and efficacy of these newer agents compared to placebo or other agents.

Methods: We conducted a systematic search utilizing MEDLINE, Embase, PsycINFO and the Cochrane Library, with the aim of identifying all RCTs comparing newer atypical antipsychotics approved by the FDA since 2002 (including brexpiprazole, cariprazine, lurasidone, iloperidone, asenapine, paliperidone and aripiprazole) with placebo or another comparator, in the treatment of any phase of bipolar disorder (including mania, depression or mixed episodes while used as an acute or maintenance treatment) in older adults ( $>65$ years). We intended to assess outcome criteria of change in mood scores, proportion of participants achieving a priori criteria of remission or of response (e.g. $50 \%$ reduction in a mania or depression rating scale), and all reported adverse effect measures across the arms.

Results: We found no RCT data on any of the examined agents. Hence we changed our search criteria to include studies with a lower age cut-off (55 and above), inclusion of post-hoc studies and changing our approach to a narrative review.

Conclusions: Two post-hoc studies on lurasidone suggest its reasonable safety and efficacy profile in the acute and maintenance treatment of OABD. Further pharmacoeconomic evaluation of lurasidone needs to be conducted. Other newer antipsychotic agents need further investigation.

\section{Exercise Intervention in a Psychiatric Outpatient Clinic}

Sivan Klil-Drori, Allana Goodman, Sophia Escobar, Marilyn Segal, Karl Looper, Soham Rej

Background: Exercise has wide range of health benefits, more than any other single intervention. It improves depression and anxiety symptoms, attention and other cognitive functions. Regular exercise is recommended for common chronic illnesses, such as diabetes, hypertension, coronary heart disease, osteoporosis, sleep and more. Benefits to exercise include low cost, social interactions, no drug interactions or drug metabolism, positive side effects (as improved self-esteem, confidence, physical health) and positive stigma. However, research is required to examine the effects of exercise in older adults with mental illness, and how to tailor exercise to this population.
Methods: Objectives: To examine whether exercise, and which dose, correlates with improved well-being of older adults with mental illness. To examine whether exercise participation correlates with satisfaction and adherence to psychiatric treatment.

Hypothesis: 1. Practicing exercise could improve patient's well-being. 2. Exercise group in outpatient clinic could enhance satisfaction from psychiatric treatment and improve adherence to it.

Population: Older adults aged $>60$ with any type of mental illness, who are followed at the tertiary-care ICFP Geriatric Psychiatry Clinic, Jewish General Hospital, Montreal.

Intervention: Group exercise of 50 minutes twice a week, for 12 weeks, at medium intensity which gradually increases. Groups included 6-12 participants every 3 months and led by an exercise instructor.

Results: We will present results on approximately 15-20 patients.

Conclusions: Discussion: Exercise could potentially help number of outcomes for older patients with mental illness. Our results and future research in this field will help establish an evidence base to tailor this potential intervention to vulnerable older adults with mental illness.

\section{Towards Prevention of Mild Cognitive Impairment in Older Adults with Depression: A Longitudinal Analysis of Potentially Modifiable Risk Factors}

Damien Gallagher, Alex Kiss, Krista Lanctot, Nathan Herrmann

Background: Late life depression has been associated with increased risk of mild cognitive impairment (MCI) and dementia. Predictors of increased risk are incompletely understood. Identification of potentially modifiable risk factors could facilitate prevention of MCI and dementia. We aim to determine which clinical characteristics are associated with increased risk of MCI or dementia among older adults with depression and normal cognition at baseline.

Methods: Using data from the National Alzheimer's Coordinating Centre study participants with normal cognition and clinically defined depression were followed until development of MCI or dementia.

Results: 2655 study participants were followed for median duration of 41.8 months. 586 (22.1\%) developed either MCI $(\mathrm{n}=509,19.2 \%)$ or dementia $(\mathrm{n}=77,2.9 \%)$. In bivariate survival analyses cognitive decline was associated with age, sex, 
education, baseline cognition \& several potentially modifiable risk factors including: vascular risk factors, hearing impairment, B12 deficiency, active depression within the last 2 years and increased severity of depression. In a multivariable survival analysis (HR, 95\% CI) only age $(1.07,1.05-1.08)$, female sex $(0.72,0.59-0.88)$, education $(0.96,0.93-0.99)$, baseline cognition $(0.87,0.82-0.93)$, active depression within the last 2 years $(1.41,1.15-1.74) \&$ severity of depression $(1.05,1.02-1.09)$ remained significantly associated with development of MCI or dementia.

Conclusions: Development of MCI is associated with several potentially modifiable risk factors in older adults with depression. Future studies should determine whether active management of risk factors could reduce incidence of $\mathrm{MCI}$ in this vulnerable population.

\section{The Fountain of Health Wellness App: Inviting and} Supporting Health Behavior Change

\section{Beverley Cassidy}

Background: Health apps are increasingly available to support health behavior change. Yet few apps are specifically designed and tested or use in clinical settings and to provide encouragement based on cognitive-behavioral principles for effective health change. The Fountain of Health Wellness App is designed to be used in frontline care settings and has been beta tested in primary care for usability and helpfulness in activating and supporting patient health goals during regular office visits.

Methods: In 2017 The Wellness App was designed and beta tested to help with in-office health goal setting for frontline care. 4 primary care clinicians tested the app with 21 subjects who set goals in one of three key health behaviors : physical activity, brain challenge and social connection. Goals were set and followed over a 4 week period between office visits using the Wellness App both in office and at home to log goals and to provide encouragement for goal adherence in between office visits.

Results: Health goals set using The Wellness App achieved an $86 \%$ rate of success-partial, complete or exceeded over a 4 week period. The app was found to be helpful in setting and maintaining goal adherence by most patients and was rated as easy to use in the office by most clinicians. Difficulties noted in using the beta version of the App were several technical glitches which have been ironed out with the newest version of The Wellness App.

\section{Conclusion}

The Wellness App is an effective way to introduce and support health goal setting and adherence both during and in between clinical contacts. Pilot results confirm the app is easy use and helpful for clinicians and patients alike. The revised Wellness App is available for use in health goal setting via a log-in site on the Fountain of Health website: www. fountainofhealth.ca.

\section{\#CAGPCCSMH2018: Twitter for Education and Advocacy in Seniors Mental Health}

Amanda Canfield, Crystal Zhou, Andrea Iaboni

Background: Twitter is an online social networking service that serves as a platform for users to share thoughts, news, information, videos, and pictures. Twitter is designed for and used as a vehicle to share ideas and have online conversations ${ }^{1}$.

Many professional organizations have been using Twitter to engage delegates during conferences to allow real-time conversation on content, networking amongst attendees, and involvement of individuals unable to attend in person. The Canadian Geriatrics Society (CGS) conducted a survey following the 2015 Annual Scientific Meeting (ASM) to evaluate and describe the impact of Twitter during the conference. The majority of respondents reported that the conference hashtag (\#CGS2015) was useful ${ }^{2}$. Themes identified regarding the impact of Twitter at the conference included resource sharing, facilitation of interaction with other participants, connections nationally and internationally, and allowing for extension of discussion regarding the sessions ${ }^{2}$.

In 2018 the Canadian Academy of Geriatric Psychiatry opened a Twitter account with the handle @CAGPsych. The ASM planning committee created an official hashtag (\#CAGPCCSMH2018) for use during the 2018 ASM to augment participation, disseminate knowledge and resources, advocate in areas of seniors mental health, and facilitate networking. This interactive workshop will assist attendees with learning the basics of Twitter as well as advanced functions for active Twitter users. This will include both an expert panel that will describe their experience with Twitter as well as the benefits it has provided for their careers and concurrent hands-on workshops to enhance learning.

\section{Methods: N/A}

\section{Results: N/A}

\section{Conclusions: N/A}

References: Forgie S.E., Duff J. P., \& Ross S. Twelve tips for using Twitter as a learning tool in medical education. Med Teach.2013;35(1):8-14

Cheung B., Wong C.L., Gardhouse A. et al. (in press). \#CGS2015: An evaluation of Twitter use at the Canadian Geriatrics Society Annual Scientific Meeting. Can Geriatr J. 
Practical Strategies of Interdisciplinary Care of Behavioral Disturbances of Cognitively Impaired Older Adults in Acute Care Hospitals

Kiran Rabheru, Laura Wilding, Vera Hula, Margaret MacKenzie Neil, Nadine Sebahana

Background: The overlap of physical and mental illness in older adults has a significant negative impact on our health care system. This is especially true in the acute care hospitals, where behavioral and mental health factors result in longer length of stay, high ALC rates, poorer patient function compared to baseline, higher rate of institutionalization and poorer quality of life. Medically or surgically ill older adults with cognitive impairment in acute care often experience behavioral disturbance and their care can be extremely challenging.

Methods: The Geriatric Psychiatry Behavioral Support Team (GPBST) has existed at The Ottawa Hospital for 5 years. It consists of 3 behavioral support nurses, who provide front line assessments and interdisciplinary tailored team care planning. Supported by a geriatric psychiatrist and an APN manager, they work closely with the referring team to enhance their capacity to manage patients, communicate with family, and community agencies.

Results: This workshop will be presented by the GPBST to discuss strategies used in assessment, triaging, and interdisciplinary team care planning using real cases. Both non-pharmacological and pharmacological strategies will be discussed. Challenges cases and innovative ways to manage them will be presented in an interactive manner.

Conclusions: Our team's goal is to improve the quality of life of older adults with cognitive impairment and behavioral disturbances in acute care and to transition them smoothly back to the community. It is also our goal to help the attendees of this workshop to enhance their skills at doing the same.

\section{Developing Interactive Modules for Geriatric Psychiatry Curriculum}

Terry Chisholm, Cheryl Murphy

Background: Geriatric Psychiatrists are challenged to provide curriculum for small groups of subspecialty residents in a year-long program. Traditional didactic series are often not the most practical solution. Evidence supports using Adult Learning principals and self-directed learning to provide engaging opportunities for the subspecialty resident to learn.
Methods: At Dalhousie we have developed an online curriculum and have used the program Articulate to make the modules interactive and engaging.

Results: We plan to explore the rationale and techniques used to create the online learning modules, while encouraging participation of the audience. We will demonstrate elements from our curriculum, and employ small group exercises and large group discussion to promote audience participation.

Conclusions: The participants will be expected to explore how to provide an engaging and interactive curriculum for their residents using the principals and techniques discussed.

\section{Providing Telepsychiatry Services: A Survey of Members of the Canadian Academy of Geriatric Psychiatry}

David Conn, Robert Madan, Marie-Andree Bruneau, Cindy Grief, Catalina Lopez de Lara, Bilal Yousuf

Background: Telepsychiatry has been reported to be feasible in the delivery of mental health series across an array of populations, including older adults in varied clinical settings. Many areas of Canada have very limited access to psychiatric care and virtually no access to geriatric psychiatry. The purpose of this survey was to a) determine the current level of provision of telepsychiatry by Canadian Geriatric Psychiatrists b) attempt to delineate areas requiring increased services and c) understand barriers to the development of increased services.

Methods: Data was collect through an online survey. The survey was sent out to the 209 full members of Canadian Academy of Geriatric Psychiatry (CAGP) via email following approval by the CAGP Board. Participants were invited to complete the electronic form of the survey either in English or French.

Results: Fifty-one physicians completed the English version and 10 physicians completed the French version for a total response rate of $29.2 \%$. Geriatric telepsychiatry services were provided by $46 \%$ of responders. Sixty-five percent of responders who provide telepsychiatry services provided less than 5 hours on average per month. Seventy-seven percent of those who answered the question said they are interested in providing telepsychiatry services in the future. Ninety percent of responders believe that their province could benefit from an increase in geriatric telepsychiatry services.

Conclusions: The authors suggest that strategies should be developed to encourage the development of increased telepsychiatry services for older Canadian adults. 


\section{Sexual Orientation and Gender Identity Matter: The Case for Inclusive Mental Health Practices with Older Adults}

Kimberley Wilson, Arne Stinchcombe, Kathy Kortes-Miller

Background: This presentation draws on data from three research projects focused on the diverse experiences of aging for older adults who are members of the lesbian, gay, bisexual and transgender (LGBT) communities. As Canada's population is aging it is imperative to consider the diverse experiences of aging, including those who are part of the LGBT community who have lived through unique social and historical context (e.g. homosexuality as a mental illness and/or a crime). Yet, these factors are rarely embedded into training and education programs, resulting in a mismatch in knowledge of providers and needs of older LGBT adults.

Methods: Thirteen focus groups were conducted with older adults across Canada. Participants were aged 60 and above and self-identified as a member of the LGBT community. Audio was transcribed and analyzed using grounded theory methodology. Additionally, baseline data from the Canadian Longitudinal Study on Aging (CLSA) was analyzed to compare self-reported mental health indicators by sex and sexual orientation.

Results: Participants spoke to the importance of having healthcare providers who acknowledge and recognize their sexual orientation and gender identity and are aware of their unique aging needs. Fears about being re-closeted in order to receive care and the resulting mental health impacts were key findings. Participants in the CLSA tended to report high self-rated mental health, however there are disparities between self-reported depression and anxiety between sexual minorities and their heterosexual peers of the same sex.

Conclusions: Sexual orientation and gender identity matter when it comes to providing mental health services and support for older adults. Health and social care providers should work consider the unique social and historical contexts of sexual minority older adults.

\section{An Immersive Simulation Experience to Build Empathy for Geriatric Patients}

Petal Abdool

Background: As we face the impending "silver tsunami", it is imperative for health professionals to gain proficiency in working with elderly patients who suffer from co-morbid physical and mental illness. A key challenge lies in developing and supporting continued empathy towards this stigmatized population. Simulation can provide standardized experiential learning opportunities to address this critical gap.,
Methods: We designed a simulation experience where participants first take on the perspective of a geriatric patient with advanced mental illness through a narrative writing exercise and then physically inhabit this role by wearing an aging simulation suit to perform common tasks (e.g., reviewing and signing a consent form, putting on a gown). Participants then engage in reflective observation about their experience through an additional narrative writing exercise and a facilitated debriefing session. Individual interviews to be conducted 3 months post-intervention will explore the impact of the intervention on their clinical practice.

Results: Data collection will begin in June 2018 with an initial target population of psychiatry residents, which will then be extended to inter-professional students and teams. Our program evaluation will adopt a mixed qualitative and quantitative analysis approach to explore both process and outcome dimensions related to empathy building.

Conclusions: We anticipate that participation in this experiential learning opportunity will result in increased empathy for geriatric patients and also serve to stimulate meaningful discussion regarding the challenges faced by this vulnerable population in the healthcare system.

\section{Integrated Mental and Physical Healthcare of Older Adults: How Do We Deliver the Goods?}

Eileen Burns, Samir Sinha, Kiran Rabheru, Monica Bretzlaff, Simone Powell

Background: In keeping with this year's theme, "Integrated Mental Health and Medical Care for Older Adults: Bringing Science and Practice Together," this engaging panel of keynote speakers and opinion leaders in Canada will serve to highlight and elaborate on our conference theme.

Methods: Team-based approaches offering integrated holistic care is delivered in collaboration with older adults has the potential to improve outcomes for patients and their families. Dr. Sinha, Dr. Burns, Dr. Rabheru, Dr. Conn, Ms. Monica Bretzlaff and Ms. Simone Powell will engage in an interactive discussion focused on how to optimize this potential and "deliver the goods" in inter-professional practice. Their perspectives on the key element that fosters synergistic collaboration among primary care, specialty care, allied health and lived experience care partners will be offered. Evidence-based methodologies for practical application and real-world influence of age-friendly initiatives, ageism, prevention, health promotion and prevention will be interwoven throughout this collaborative session.

Results: The discussion panel members will offer their "best idea" for promoting inter-professional healthcare integration 
from their points of view. A question and answer discussion period with the audience will allow for a meaningful knowledge exchange about this year's conference theme in practical terms - on how we can "deliver the goods" for older adults.

Conclusions: A panel discussion about Integrated Mental, Physical and Behavioural Healthcare for Older Adults \& Their Care Partners meaningfully elaborates the conference theme and how to reach the full potential of an inter-professional care approach to improve health outcomes.

\section{There's No Place Like Home - Meeting the Needs of Nova Scotia's Aging Population}

Susan Stevens, Gerry Hamilton, Patricia Baker, Kathy Bonner

Background: Between 2007 and 2013 the number of people waiting for a long term care bed in Nova Scotia increased $267 \%$ and wait times almost doubled. Yet the $75+$ population only increase $7 \%$ over this time. The average length of stay in Nova Scotia nursing homes increased from 2.2 years to 2.9 years. Every year between $400-600$ people on the wait list refused a bed offer because they were not ready for placement. Wait lists for home support grew to almost 900 people waiting for over 12,000 hours of care in early 2015 . With one of the oldest populations in the country, Nova Scotia's continuing care system was under strain.

Methods: In 2012/13, Nova Scotia began rebuilding capacity within continuing care through an intentional focus on a new Home First approach, including improved integration and collaboration with acute care services. Foundational to this approach is patient and family centred care through a meaningful understanding of what and who is most important to the individual. Policy and practice changes were also made to improve access to services through reducing and eliminating waitlists in home care and long term care.

Results: Patients whom physicians, health care professionals and in some cases family thought required long term care have successfully transitioned home. One such patient journey will be shared detailing how after less than one week in hospital she was referred for nursing home placement and how she and her allies made home first a reality where a year after her admission to hospital, she continues to live independently with supports.

More patients are being discharged home from hospital and fewer patients are being transferred from hospital to long term care. Provincially placements from hospital have decreased from $50 \%$ of total placements to $40 \%$ of placements. Average lengths of stay in home care are increasing.

Conclusions: Taking a Home First approach benefits both individuals and families in achieving their desired outcomes, improves access to services across the continuum and ensures appropriate health care allocation. In this symposium Nova Scotia's Home First transformation will be shared both at the patient/family level through the firsthand account of one patient's personal journey from hospital to home and at the system level through key performance indicators and health quality improvement methodologies. The critical role physicians and allied health professionals play in both these stories will be discussed.

\section{The CARER Tool: A Novel Non-Pharmacological Intervention for Family Caregivers}

Robert Madan, Kenneth Schwartz

Background: Responsive behaviours in dementia are associated with poor outcomes for the person with dementia and caregiver burnout. Various non-pharmacological approaches currently exist (e.g. PIECES, DICE), but it is clear that caregivers continue to need tools to manage responsive behaviours. The CARER tool was studied in long term care with formal caregivers and the results were promising. The tool was simplified and is being studied with family caregivers using educational videos. The goal of the workshop is to review the current evidence and to teach participants how to use the new CARER tool for caregivers to manage responsive behaviours.

Methods: A series of educational videos were created using volunteer actors to demonstrate how family caregivers could use this new tool to manage responsive behaviours. The videos are being shown to family members of persons with dementia. A mixed method approach using qualitative and quantitative approaches is being employed to evaluate caregiver burden, satisfaction, feasibility and utility of the CARER tool.

Results: By the end of the session, participants will be able to list evidence-based approaches to responsive behaviours in dementia, describe the theoretical basis for the CARER tool, and list the steps involved in managing responsive behaviours using the CARER tool. It is anticipated that the results of this current study will demonstrate the utility, feasibility and effectiveness of the CARER tool.

Conclusions: The CARER tool is a novel intervention for caregivers for persons with dementia. It is anticipated that family caregivers will find the tool easy to use and of great utility.

\section{Autism in the Elderly: Addressing its 'Existence' and Recognition of Features and Management}

Shabbir Amanullah, Sameh Hassan, K. S. Shivakumar, P. Krishna 
Background: Autism Spectrum Disorders (ASDs) is an umbrella term for autistic disorder, Asperger's disorder, and pervasive developmental disorder. It is the second most common severe developmental disability in the United States after Intellectual Disability (Mental Retardation) .

An English study stated that the prevalence of ASDs in older age groups was $1.1 \%$ (ages $16-44$ ), $0.9 \%$ (ages $45-74$ ), and $0.8 \%$ (ages 75 and older). In the USA alone, the increase of population numbers will lead to a prevalence of 700,000 subjects with ASD who are older than 65 by 2030 . This is in part a result of the significant increase in life expectancy that occurred over the last few decades.

Another English study suggests that in 2026, in the UK alone, there will be approximately 170,000 older individuals who will need some type of constant care. This number may actually be a false estimate and the real number could almost be double the figure as ASD was found to be an underdiagnosed disease within their service system . Currently there are an estimated 8 million people in Europe with ASD, 20\% of which are older than 65 years of age.

Methods: We reviewed existing literature and key articles on Autism Spectrum Disorder in adults and the elderly. We searched Google Scholar, PsychINFO, Medline, Cochrane, and CINAHL. We used for our search combinations of the following keywords: "Autism Spectrum Disorder", "ASDs", "Adults", "Elderly", "Screening", "Diagnosis", "Treatment", "Manifestations", and "Interventions". A study was considered eligible for inclusion if the disorder being addressed fulfilled DSM-IV criteria for ASDs. Overall, 40 studies were reviewed.

Results: Many screening tools have been developed for screening children with suspected ASD, but they can not be applied in later life. There are three screening instruments that exist for adults. The Autism-Spectrum Quotient (AQ) was developed by Baron-Cohen and their team in 2001 and has 50 items to be rated by the patient. It assesses 5 main fields; social skills, attention switching, attention to detail, communication, and imagination. Since many patients can not recognize their symptoms, suggestions to improve its validity have been made by also having family members and close friends complete the Autism Questionnaire for relatives (AQ-R)11, . This tool still needs testing for effective use in the future, although it has been recommended by a study done in 201011.

Conclusions: The ability to recognize ASD is critical to effective patient care. One needs to be able to create a positive learning environment but also create an effective strategy to identify, teach, engage and communicate with patients with ASD.

The lack of input will only contribute to hardship for the patient.

\section{Provision of Geriatric Psychiatric Care in a Rural Primary Care Practice Utilizing Allied Health Care Professionals}

\section{Heather Haworth, Lisa Seymour}

Background: New Glasgow is a small rural town in Nova Scotia. Like most rural areas in the Maritimes it has a growing geriatric population. In 2008 a collaborative primary care practice was established consisting of 5 primary care practitioners, a nurse care practitioner, 2 family medicine nurses, part time dietician, social worker and pharmacist.

It is not uncommon for a patient or family member to complain to their doctor problems with memory.

With the assistance of the urban geriatric psychiatric team in Halifax, the social worker was trained to assess cognitive functioning. This training was utilized to assess patients in their homes sometimes with another allied health care professional from the practice.

Methods: Monthly telepsychiatry sessions supported continuing education. Home visit assessments included interviews with the patient and family member. Standardized cognitive, depression and functional assessment tools were administered. The results were then conveyed to the primary care physician.

Results: Utilizing allied health care professionals for the assessment of patient functioning in their homes allowed for a much more thorough assessment than could be provided in the clinical office of the primary care physician.

- It allowed early intervention when cognitive impairment was noted.

- Competency was better assessed.

- The patient was more comfortable reducing performance anxiety.

- More often than not the patient was assessed as normal or mild cognitive impairment contradicting the suspected dementia diagnosis .

Conclusions: With training, a rural primary care practice can provide quality and expedient geriatric psychiatric assessment and care through the use of allied health care clinicians

\section{Joint Impact of Frailty and Depression on Cognition}

Melissa K. Andrew, Judith Godin, Ravi Bhat

Background: Links have been established between frailty and cognition and between depression and cognition, but little research exists into how these two factors may work together to influence cognition. Further, there is debate about 
how different patterns of depression (e.g., late-onset versus long history of depression) may be differentially associated with cognitive impairment.

Methods: In secondary analysis of Waves 1, 2, 4, and 5 of the Survey of Health Ageing and Retirement in Europe (SHARE), 1862 participants age 65 years or older were categorized as never-depressed (60.6\%), past depression only $(19.9 \%)$, current depression with onset at age 65 or later (15\%), and past and current depression (9.2\%). Latent variable growth curve modeling was used to assess whether the relationship between frailty and cognition varied based on depression category.

Results: After controlling for age, sex, social vulnerability, education, and alcohol consumption, frailty was associated with a steeper decline in verbal fluency in currently depressed participants compared to never depressed participants, $b=$ $0.24, \mathrm{p}<.01$. Frailty was associated with a steeper decline in instant recall in past and currently depressed participants compared to never depressed participants, $b=-0.61, p<.05$. Frailty was associated with a steeper decline in delayed recall in currently depressed participants, $b=-0.05 p<.05$ and those with past and current depression, $b=-0.07, p<.05$ compared to never depressed participants.

Conclusions: We found an association that the established connection between frailty and cognition varied depending on the history of depression, suggesting time of depression onset is also an important consideration.

\section{Capacity Assessment In Older People: Interactive Case Workshop}

\section{Karen Reimers}

Background: As the population ages, clinicians are increasingly faced with questions about the mental capacity of elderly patients, including the elderly patient's capacity to consent to or refuse treatment, drive, manage independently at home, and more. These scenarios are commonly seen by general and geriatric psychiatrists as well as primary care providers and advanced practice clinicians across many settings including outpatient clinics, hospitals, nursing homes and others. The consequences of determining a patient's mental capacity can be far reaching. Elderly patients and their families can benefit from a practicing clinician's competent assessment of the elderly person's mental capacity when indicated.

Methods: This interactive case-based workshop will engage participants in a lively discussion of common clinical scenarios involving assessment of mental capacity in the elderly. Each case is based on actual clinical experience with elderly patients in a general psychiatric practice. Cases are selected to reflect common scenarios that clinicians are likely facing in their daily practice, whether they work in a primary care or psychiatric specialist setting.

Results: We will identify the most common areas of clinical difficulty for practicing clinicians. The range of cases will include assessment of capacity to make medical decisions, manage finances, driving capacity, the role of psychiatric and medical disorders affecting mental functioning, and more.

Conclusions: Following each case discussion, we will review pertinent clinical and ethical dimensions for that case. We will highlight recommendations and practical strategies for clinicians to implement in their practice. References for each case will include peer reviewed literature in this evolving field.

\section{Help Us Build the Atlantic Seniors Mental Health Network (ASMHN): Bring Your Boldest Ideas!}

Keri-Leigh Cassidy, Shabbir Amanullah, Beverley Cassidy, Debbra Cyr-Lebel, Sarah Krieger-Frost, Sarah Thompson, Dean Gambin, Katie Aubrecht, Wendy McVeigh

Background: The Atlantic Seniors Mental Health Network (ASMHN) was officially launched in 2010 with the mandate to build and support a stronger community of practice in mental healthcare of older adults in Atlantic Canada.

Methods: A 2018 environmental scan was conducted to assess human resource assets and liabilities in Atlantic Canada for seniors' mental healthcare. Representatives from the four Atlantic Provinces were canvassed to assess the extent of vulnerabilities, including scarcity of specialized geriatric resources, need for educational support and greater interprovincial collaboration in the health and continuing care sectors.

Results: The ASMHN aimed to redress the above vulnerabilities in 2018 with two initiatives: 1) a mass e-list to broaden ASMHN membership and improve interprovincial communication and 2) develop a "clearing house" of webinar /CME events for ASMHN affiliates and front line care providers in Atlantic Canada. This workshop will engage participants to consider directions forward.

Conclusions: The ASMHN is an opportunity to build a stronger community of practice in mental healthcare in Atlantic Canada. The network hopes to do this through networking, and educational offerings for frontline care. Bring your boldest ideas for the ASMHN to this workshop! Cross-discipline discussion and solutions will be needed to grow an effective Atlantic network. 


\section{A Case of Mania Following Stroke}

Elyse Ross, Lauren Mussen, Amer M. Burhan

Background: The prevalence of mania in the post-stroke setting is low, and often may be a misdiagnosed delirium. We describe the case of a 71-year-old man with new onset mania following an occipital and cerebellar embolic stroke in 2014 and TIA in 2016. The patient had no psychiatric or substance use history. His medical history included prostate cancer with radical prostatectomy, mild chronic kidney disease, dyslipidemia, and deep vein thrombosis. Following the stroke in 2014, he experienced mildly increased talkativeness and reduced ability to participate in conversation. These changes remained stable until following the TIA in spring 2016. He then presented with new-onset affective changes alternating between extreme irritability and euphoria, hyperactivity, distractibility, disinhibition, hypersexual behaviour, thought disorder, and psychosis.

Methods: Investigations included MRI head in 2015 revealing bilateral occipital lobe infarcts, left cerebellar infarct, remote left thalamic lacunar infarct and focal cortical atrophy of the rostral frontal lobe and parietal lobes. Cognitive testing during the manic episode revealed MoCA score of 26/30. He required involuntary admission to a peripheral psychiatric facility and was treated with carbamazepine, trazodone, and cyproterone, with no effect. He was transferred to a tertiary psychiatric facility and initiated on olanzapine and lithium.

Results: The patient responded well to combination therapy, and was discharged home one week later. He remains stable one year following discharge.

Conclusions: Mania may present as a complication of stroke. Our case suggests that timely recognition and treatment can improve patient outcomes.

Convulsive Neurostimulation Therapies in Parkinson's Disease with Depression: Protocol for a Prospective, Open-Label Clinical Trial Comparing Outcomes of Electroconvulsive Therapy and Magnetic Seizure Therapy

Nicholas J. Ainsworth, Fidel Vila-Rodriguez

Background: Parkinson's disease (PD) is a neurodegenerative condition comprising a significant source of morbidity in older adults. In addition to the motor symptoms of Parkinson's disease, increasingly recognized are the so-called non-motor syndromes of Parkinson's disease: among these, Parkinsonian depression (Pd) and Parkinson's disease with dementia (PDD), are arguably most influential on clinical course and quality of life for PD patients. Limited clinical evidence exists regarding the prognostic impact of $\mathrm{Pd}$ and its treatment on developing PDD or another major neurocognitive disorder. Electroconvulsive therapy (ECT) is a form of neurostimulation considered the gold standard treatment for severe and treatment-resistant Major Depressive Disorder (MDD), for which substantial observational evidence also exists in the setting of Pd. ECT has also been reported to transiently improve motor symptoms in PD. Magnetic seizure therapy (MST) is an experimental noninvasive neurostimulation treatment, which uses magnetic induction to induce a current in the brain which elicits a generalized tonic-clonic seizure. First used for treating MDD, MST is a plausible therapeutic candidate for Pd patients, which carries the appeal of comparable efficacy to ECT while having a favourable neurocognitive side-effect profile.

Methods: This pilot, prospective study (target $n=20$ ) will be conducted at the University of British Columbia Hospital. Its primary aim is to test a noninferiority hypothesis for MST compared with ECT with respect to treatment of depressive symptoms in Pd patients. Secondary outcomes include effects on motor symptoms and changes in neuropsychological performance during the treatment and follow-up phase. Investigational measures including EEG and f/MRI will be performed to identify potential predictive biomarkers of treatment response. In the follow-up phase, longer-term neurocognitive outcomes including the incidence of new MCI and major neurocognitive disorder diagnoses will be measured.

\section{Evaluation of Anticonvulsants Use in Management of Alcohol Withdrawal Syndrome and Long-term Medication Management of Alcohol Use Disorder in Older Adults}

\section{Stefanie Montogmery}

Background: Alcohol withdrawal syndrome (AWS) occurs after discontinuation of excessive alcohol consumption. The geriatric population is at higher risk for AWS complications due to a greater prevalence of comorbidities, a longer duration of alcohol abuse, baseline cognitive deficits, and sensitivity to treatment. Benzodiazepines remain to be the mainstay therapy to reduce the severity of symptoms and incidences of seizures and delirium tremens. Vancouver General Hospital (VGH) has pre-printed order sets that follow the Clinical Institute Withdrawal Assessment-Alcohol revised (CIWAAr) format to provide symptom-triggered benzodiazepine therapy. Ajunctive anticonvulsants may also be prescribed to reduce symptom severity. The use of anticonvulsants in the elderly population for alcohol withdrawal has limited evidence despite being potentially efficacious and safe while providing mood stabilizing or anxiolytic effects. Inpatient management often focuses on the withdrawal symptoms 
and it is unclear how much attention is given to the longterm treatment of alcohol use disorder using agents such as disulfiram, naltrexone and acamprosate.

Methods: A retrospective chart review of patients admitted to medicine units that are started on the AWS protocol between June 2013 - June 2018. The primary objectives are whether adjunctive anticonvulsants for AWS or long-term treatments for alcohol use disorder are initiated. Secondary objectives include adverse events secondary to the treatment of anticonvulsants and benzodiazepine therapy and a quality assurance of the pre-printed AWS protocol that is currently in use at $\mathrm{VGH}$.

\section{Cardiac Monitoring: Should Specific Protocols Be Developed for Older Adults with Schizophrenia}

\section{Catherine Bobek}

Background: Prolongation of the corrected QT interval (QTc) raises concerns in clinical practice as it can lead to life threatening arrhythmias. Many psychotropic drugs can prolong the QTc interval and potentially lead to sudden cardiac death. Despite the risk of cardiac complications, drugs remain the cornerstone of treatment for mental illnesses such as schizophrenia and mood disorders. The goal of this study is to make recommendations for best practice to facilitate appropriate medical care for this patient subgroup population.

Methods: This study is a population-based cross-sectional study using administrative databases at the Institute for Clinical Evaluative Sciences at Queen's University. The study population will include older adults, ages 66 years and older identified as having schizophrenia or a related psychotic disorder using a validated algorithm between 2008 and 2016 in Ontario, Canada. Individuals will be followed from the date of cohort entry until their first new prescription of one or more antipsychotics in the known risk of QTc prolongation. The frequency of ECG monitoring in the 90 days immediately preceding the date of the first new high-risk antipsychotic prescription and 90 days immediately following the prescription will be determined. The characteristics of individuals who did or did not have any ECG monitoring in the time period surrounding new prescription of high-risk antipsychotic medications will be compared on their demographics, medical history, psychiatric history, prescribing of concomitant medications including other QTc prolonging medications, frequency of other QTc risk factors and health service use. Multivariate logistic regression will be used to determine the odds ratio and $95 \%$ confidence intervals.

\section{How Interprofessional Collaboration Promotes Resident Engagement in a Rural, Long-Term Care Setting}

Yvonne Tieu, Stephanie Mattson, Chad Bauld, Tina BoydFuller, Jacquelyn Levy

Background: Older adults with disabilities living in longterm care settings have complex and varied needs, often requiring collaboration among multiple professionals. Approaches and strategies to promote meaningful engagement and activities typically require teamwork and creative application of best practice approaches.

Methods: The poster will review the current literature on best practices and evidence-based approaches to promote meaningful engagement of residents in long-term care centres. In particular, research discussing the importance of resident engagement, quality of life, and use of assistive technology will be highlighted. Strategies to promote resident engagement through interprofessional collaboration involving Occupational Therapy, Psychology, Social Work, Assistive Technology, Nursing, and frontline staff members will be reported.

Results: Collaborative interprofessional implementation of meaningful activities and assistive technologies will be discussed through case examples of older adults with disabilities and/or mental health issues. Of focus will be the use of iPads for leisure, social, and educational activities. Challenges related to interprofessional collaboration and application of best practices will be reviewed. Considerations for the generalization of activities and assistive technologies to other long-term care settings will also be discussed.

Conclusions: The methods used to promote resident engagement through interprofessional collaboration (e.g., Occupational Therapy, Psychology, Social Work, Assistive Technology, Nursing, and frontline staff members) in a rural, long-term care setting for older adults with disabilities will be presented.

\section{Geriatric Telepsychiatry to the Home-Remote Delivery of Psychogeriatric Care in Toronto, Canada}

Christopher Kitamura, M. Uri Wolf, Haddas Grosbein, Jahnel Brookes, Anna Theresa Santiago, Rosalind Sham, Sarina Trac, Jordanne Holland

Background: Telepsychiatry has had an increasingly important role in providing access to mental health services. Geriatric psychiatry outreach services provide multidisciplinary home visits to frail elderly individuals. Travel time by psychiatrists limits the availability of home visits. The present project pilots telepsychiatry visits involving one member of the team, or a retirement home staff member, assisting the 
patient in their home as they communicate with a geriatric psychiatrist, who will conduct assessments from their office.

Methods: A convenience sample of patients was obtained from 2016-2017. Upon receipt of referrals, patients were assessed for eligibility criteria and approached by study personnel for consent (caregivers consented on behalf of incapable patients for survey completion). Eligibility criteria included English speaking, absence of health problems precluding telepsychiatry assessment, home environment safe to enter; and patient not requiring urgent assessment where delays may cause harm. Patients ineligible for the study were offered the standard procedure of an in-person assessment.

Results: 21 unique study patients were assessed in a total of 11 initial visits and 19 follow-up visits. Six caregivers and 7 patients provided feedback. Three psychiatrists provided assessments. Mean patient age was 84.0 years and $71 \%$ were female. Patients lived a mean distance of $4.8 \mathrm{~km}$ from Baycrest. $100 \%$ of patients and caregivers providing feedback replied yes to the question, "I was satisfied with my videoconferencing visit." Qualitative feedback from participants: 2 psychiatrists, 7 case managers, 3 caregivers, 1 patient, and 3 non-OTN staff were collected after the study evaluation period.

Conclusions: Telepsychiatry presents a novel tool in our urban geriatric psychiatry outreach team. Quantitative and qualitative data reported speak to the feasibility and satisfaction of telepsychiatry among participants. Future comparisons between telepsychiatry and home visits as to wait times, clinical outcomes, and overall capacity to provide care warrants further study.

\section{Bespoke Education - Tailoring Learning to Meet the Needs of Diverse Healthcare Professionals in Geriatric Mental Health}

Cindy Grief, Lisa Sokoloff, Alvina Anantram, Cindy Plunkett, Daphna Grossman, Raquel Meyer, Agnes Cheng Tsallis, David Conn

Background: In the marketing world, it is the norm to give individuals options for customizing their new car or suit; this made to order process is called "bespoke". We believe the content of continuing education programs lends itself to customization. Interprofessional healthcare team members may have different learning needs which can vary. Benefits of customized CE modules include improved learning outcomes, increased uptake of QI tools in clinical settings and cost-effectiveness. Previously we paired eLearning with live interactive webinars and demonstrated the feasibility of this virtual blended learning approach for delivering $\mathrm{CE}$ in geriatric mental health. Anticipating diverse learning needs of interprofessional participants is challenging.

Methods: We developed a customized eLearning module, "Depression, MAID and the Older Adult" paired with a live "ask the expert" webinar. Content was informed by bespoke needs assessments completed via surveymonkey and live webinar (to further clarify some needs). Participants were asked about inclusion of specific components and for questions they would like addressed.

Results: We hypothesized that customized education modules in geriatric mental health would improve effectiveness of learning measured by changes in knowledge, skills and attitudes.

- $71 \%(\mathrm{n}=20)$ felt live needs assessment was very useful to share thoughts/questions; $28 \%$ felt somewhat useful

- Post activity $(\mathrm{n}=31)$

- $\quad 87 \%$ respondents indicated "bespoking" enhanced learning

- $\quad 77 \%$ noted location-based cases enhanced learning

- $\quad 93 \%$ want more bespoke education opportunities

- $77 \%$ are more likely to use tools described in the module

Conclusions: Customizing education takes time but is effective in enhancing the learning experience.

\section{Utility and Feasibility of Self-Rating Scales in Geriatric Mental Health}

Cindy Grief, Daphna Grossman, Anna Berall, Sandra Gardner, Shoshanna Campbell

Background: Scales for monitoring mood, anxiety and physical symptoms have been well validated in older adults with psychiatric disorders within inpatient and outpatient settings. Older adults with mood disorders frequently endorse somatic symptoms including sleep difficulties and chronic pain which are independent risk factors for depression. The purpose of this study was to determine the feasibility of using self-rating scales for older adults in outpatient and inpatient geriatric mental health settings to monitor mood and physical symptoms.

Methods: Patients from a Geriatric Psychiatry Day Hospital and a geriatric psychiatry inpatient unit completed self-report rating scales to track mood, anxiety and somatic symptoms and their respective impact on dignity and quality of life, weekly for a median of four months. Patient demographic characteristics were collected. Data analysis included descriptive summaries including means, standard deviations (SD), percentages and correlation coefficients. 
Results: Data from 33 English speaking patients (inpatients $\mathrm{N}=17$, outpatients $\mathrm{N}=16$ ) included a mean age of 76.5 $(\mathrm{SD}=6.1), 69.7 \%$ were female and $48.5 \%$ were married. At baseline, higher GDS scores were significantly correlated with self-ratings for poor dignity and high anxiety $(\mathrm{p}<.0001)$, poor quality of life $(\mathrm{p}=.0004)$ and increased symptom burden $(p<.0001)$. Geriatric Depression Scale (GDS) scores decreased over time but less rapidly for patients reporting a higher degree of pain $(\mathrm{p}=0.05)$.

Conclusions: Self-report scales were found to be feasible and yielded important clinical information among older outpatients and inpatients with mood and related disorders. Subjective distress experienced by individuals revealed underlying pain and symptoms which were positively associated with mood.

\section{Contribution of Impulsivity to Falls Risk in People with Advanced Dementia: Assessment of a Modified Falls- Related Impulse Behaviour Scale (mFIBS)}

Caitlin Armer, M. Marcil, A. Lake, Andrea Iaboni

Background: Current falls risk assessment tools have limitations in advanced dementia: while they accurately assign virtually all individuals as high risk for falls, they are not specific enough to identify those at the highest risk and require closer monitoring. The Fall Related Impulsive Behaviour Scale (FIBS) helps to incorporate the common dementia symptom of impulsivity into the fall risk assessment. The purpose of this study is to evaluate the sensitivity and specificity of a modified FIBS tool (mFIBS) for fall risk in patients with advanced dementia.

Methods: We are completing a retrospective chart review of 100 patients admitted to a tertiary dementia behavioural care unit. We are recording their STRATIFY scores, mFIBS scores, a description of any falls events and other falls risk factors.

Results: Our preliminary analysis of the first 35 patients has identified 14 "Fallers" (40\%) and 21 "Non-Fallers" (60\%). The average STRATIFY score for "Fallers" was found to be $3.4 \pm 1.1$ and $2.3 \pm 1.0$ for "Non-Fallers". The average mFIBS score was found to be $2.1 \pm 1.3$ for "Fallers" and $1.2 \pm 1.3$ for "Non-Fallers". Following analysis of the remaining 65 patients, next steps will be to develop a Receiver Operator Curve and calculate the sensitivity and specificity for the STRATIFY and mFIBS tools, alone and in combination, in this patient population.

Conclusions: This study aims to evaluate the sensitivity and specificity of the mFIBS tool for fall risk in patients with advanced dementia, to determine if it can improve upon or add to existing falls risk assessments in this population.

\section{The Green Card: Geriatric Psychotropic Drug Reference Card}

Kathleen Singh, Terry Chisholm, David Gardner

Background: Psychopharmacology in older adults is more complicated. Reasons for this include polypharmacy, multiple medical comorbidities, and pharmacokinetic/dynamic changes with age. A nonpharmacological approach is sometimes most prudent in this population because of increased risk of drug drug interactions and adverse drug events compared to younger people. When a pharmacological treatment is used, there is often discrepancy between clinical practice and recommendations in practice guidelines. The Green Card acknowledges these challenges by attempting to consolidate pertinent clinical information onto a quick reference guide, assisting the busy clinician in their daily practice.

Methods: Information was gathered through a rigorous literature review and through both local and national expert consensus among geriatricians and geriatric psychiatrists. It attempts to bring expert opinion and practice guideline information together.

Results: The Green Card lists commonly required information for various medication classes such as recommended dosing ranges, half-lives, and $\mathrm{mg}$ /form supplied by pharmacy for antidepressants, antipsychotics, cognitive enhancers, mood stabilizers, and sedatives. It also includes pertinent clinical information including safety concerns, monitoring parameters, and contraindications. It offers both nonpharmacological and pharmacological approaches to common presenting complaints including neuropsychiatric symptoms of dementia, delirium, insomnia, and supports reducing pill burden (deprescribing). It outlines recommended actions for commonly used medications when electroconvulsive therapy is used concurrently. Finally, it lists common drug drug interactions with psychotropic medications and notable changes in the elderly that affect prescribing.

Conclusions: Its intended use will be for learners, interdisciplinary health team members, and any physician who sees older individuals.

\section{Behavioral and Psychological Symptoms of Dementia (BPSD): A Teleconsultation and Mentoring Project}

Marie-Andrée Bruneau, Nathalie Bier, Anne Bourbonnais, Stéphanie Daneau, Catherine Dubé, Caroline Ménard, Marc Bassette

Background: Most people with neurocognitive disorders will present BPSD. The Quebec Alzheimer's report disclosed 
accessibility difficulties to clinical expertise, lack of staff's training and an excessive use of antipsychotics. Many studies demonstrated the relevance of teleconsultation for assessing and treating elderly with psychiatric and cognitive disorders. However, few studies have specifically studied this technology as a way to disseminate expertise about BPSD.

Methods: A teleconsultation and mentoring service for BPSD was offered by the IUGM to a remote partner, CISSS-des Iles-de-la-Madeleine. First, the remote team underwent formal training by IUGM experts. Then, a complex BPSD case was discussed each month over teleconsultation. This project evaluated the clinical benefits (NPI-R, CGI), the implementation process (interviews, satisfaction questionnaires) and the improvement of stakeholder's knowledge (written tests).

Results: Twelve teleconsultations occurred. CGI improved in $7 / 9$ patients, NPI-R showed a decrease of $35 \%$. The remote team and caregivers were mostly satisfied with this service. The open interviews revealed that the remote team felt relieved, more competent and autonomous, that the care trajectories were clearer, that they had a better recognition and evaluation of BPSD and intervene faster. The remote team showed a global improvement in their written test results.

Conclusions: In conclusion, we saw clinical benefits as well as improvement in knowledge and competency of a remote team with this clinical and mentoring project. The different actors are satisfied with this technology. This study suggest that teleconsultation is a good way to disseminate expertise concerning BPSD and that formal teaching and mentoring aspects are essentials in the process.

\section{Development and Validation of Tamil Version of MMSE}

Kuppuswami Shivakumar, Shabbir Amanullah, Sivakumar Ramachandran, Shahul Hameed

Background: Mini-Mental State Examination (MMSE) is the most commonly used tool to assess cognitive impairments. MMSE is translated and adapted in different languages to meet the cultural needs of the population. South Asians were the largest visible minority group in the last Canadian census, accounting for $25 \%$ of all visible minorities. In the same census, there were 90,415 South Asian Canadians aged 65 and older. Among the South Asian community, Sri Lankan Tamils are a significant proportion, and the lack of availability of any assessment tool such as MMSE contributes significant under-recognition of the exact prevalence of dementia in Tamil community. Though MMSE is widely used, it is notable that availability of Tamil version MMSE is ambiguous. MMSE is available in two other common South Asian languages such as Hindi and Malayalam. Hence there is an absolute need to develop and validate Tamil version of MMSE

Methods: Develop and validate Tamil version of MMSE to be used in Tamil communities in Canada and three other countries.

Results: A multicentre pilot project is planned with experts in the field of cognitive testing and application, from Canada, Malaysia, Singapore, and India. Cultural and languagespecific modifications will be prepared based on a Delphi survey. The final translated version will be validated in native Tamil population and non-residential Tamil speaking population for its validity.

Conclusions: An early diagnosis of dementia has potential benefits for the person with dementia and their families. This would allow both family and patients to plan for their future.

\section{Informed Practice for Mental Health Care Clinicians: Developing a Specialized Problem Solving Therapy Training Program to Enhance Access to Care for Older Adults}

Lisa Van Bussell, Nicole Marlatt

Background: Problem Solving Therapy (PST) is an evidencebased, patient-centered type of informed practice that allows clients to take control of their own life problems. PST has been shown to be just as effective as pharmaceuticals in alleviating the symptoms of common mental health syndromes, such as depression and anxiety.

Methods: In London, Ontario we have created a sustainable and specialized educational training program in PST. The training program includes an eight-hour in-person didactic workshop; eight one-hour case-based learning opportunities; and, individual case supervision with a PST trainer to work towards PST certification.

Results: Starting in December of 2014, more than 35 hospital and community-based mental health care clinicians have received this specialized training. Three in-house clinicians as PST trainers have also been obtained. Acquired knowledge, perceived confidence in their skills, level of adherence to PST principles in clinical interactions, and satisfaction with the training program itself have been recorded and monitored to ensure that the clinicians enhance their knowledge in this specialized therapeutic modality.

Conclusions: We were able to successfully train a variety of clinicians including physicians, nurses, occupational therapists, and social workers, across Southwestern Ontario in an 
evidence-based therapeutic modality to care for older adults. Our trainees who work with older adults have positively received this intensive training model. Future studies will examine the impact of booster training sessions on adherence to PST principles, extending the practice to other programs, such as Stroke and Rehabilitation, and ultimately measure the impact PST has on localized access to care.

\section{Faring Well Through Life: Identification of Resilience Factors in Older Adults}

Olga Yashchuk

Background: With the ever-evolving advances in medicine, we have been able to extend our lifespan with more people than ever now being able to call themselves centenarians. According to Statistics Canada's 2016 census figures, seniors now outnumber children in Canada. Is there something to be learned from these older adults who have successfully fared through the vicissitudes of life? How can we as health care providers promote resilience in the general seniors' population and enhance the ability to age successfully on an individual level in our patients?

Methods: A literature review of the PubMed database searching for relevant articles containing terms such as "resilience," "resilience factors and older adults," and "resilience factors and aging". English language publications only, with age range of 50 years and above or younger ages if there is reference to older adults.

Results: Identification of 5-10 most salient factors that are associated with resilience in older adults.

Conclusions: Brief discussion of the factors found to be associated with resilience in older adults and possible applications in a clinical setting, including incorporating screening about resilience factors. Resilience may already be present in our patients, and strategies to identify as well as enhance resilience in the older adult population will be discussed.

\section{Enhancing Regional Geriatric Psychiatry Services - A New Model}

Carol Ward

Background: Interior Health (IH) is one of five health authorities in British Columbia. It has 5,511 permanent residential care beds in 107 facilities spread over $215,000 \mathrm{~km} 2$ in both rural and urban areas. IH faces challenges providing quality care to seniors living in residential care with complex cognitive and mental health care needs such as those with behavioural and psychological symptoms of dementia (BPSD) because of a shortage of geriatric psychiatrists and senior's mental health in reach support.

Approximately $70 \%$ of the residential care population within IH have disorders of the brain and experience neuropsychiatric symptoms such as psychosis, intrusive wandering, physical and/or verbal responsive aggressive behaviours. These challenge the skills, creativity and coping resources of front-line caregivers. A two year project, funded and coordinated in a partnership by the Specialist Services Committee of $\mathrm{BC}$ and Interior Health, highlights a collaboration between Residential Care and Senior's Mental Health and Substance Use (MHSU) Program which developed and implemented several initiatives to develop a behavioural support system and improve the care of these seniors. This project complements IH's commitment to delivering PIECES and GPA to residential care staff, polypharmacy initiative and a quality improvement project to decrease antipsychotic use for dementia in residential care.

Methods: Deploy a specialized, support team of 2.5 FTE Regional Knowledge Coordinators-Complex Behaviours (RKC-CB) using a single entry point using a direct online referral from any residential site within Interior Health. Each of the three regional RKC-CBs has a designated number of beds; however the FTE per bed differs amongst the three areas (0.33 FTE/1000 beds in Central IH, $0.74 \mathrm{FTE} / 1000$ beds in East and $0.53 \mathrm{FTE} / 1000$ beds in the West). RKC-CBs cover $1,021,2,991$ and 1,403 beds respectively.

- $\quad$ RKC-CBs build local capacity by providing consultation via telephone, select site visits, direct teaching, and coaching. Promote the P.I.E.C.E.S. TM approach, Gentle Persuasive Approach (GPA) techniques and the BC BPSD Algorithm as the core of dementia-care educational initiatives.

- Implement a standardized approach to Behavioural Care planning and documentation

- Work with psychiatrists to develop a consistent approach to site preparation for their in-reach consultation

- Host IH-wide case based inter-professional accredited Responsive Behaviour Rounds for all staff

- Deliver the accredited workshop 'Dementia-related Responsive Behaviour: Putting It All Together Using P.I.E.C.E.S.TM and the BC BPSD Algorithm' for physicians within IH;

- Develop the above workshops into online learning modules for sustainable on-going education for physicians

Results: CMAI analysis indicates that $88 \%$ of residents referred to RKC-CB improved following the recommended interventions post consultation by RKC-CB.

- Admissions to acute tertiary mental health in-patient unit from residential care have declined by $70 \%$

- Visits to ED and admissions via ED have declined by $41 \%$ 
- "Unsafe Behaviours", including aggression trends show improvement in all types of event: "No harm" events increased by $35 \%$ and mild and moderate harm events declined by $47 \%$

- $61 \%$ of attendance who participated in Rounds indicated new or enhanced knowledge for working with residents exhibiting complex behaviours.

- $94 \%$ of physicians who attended education workshops on "Dementia-related Responsive Behaviours Putting It Altogether Using P.I.E.C.E.S. ${ }^{\mathrm{TM}}$ and the BC BPSD Algorithm".

- Estimated cost avoidance due to the reduction of acute care visits/admissions is $\$ 760,164$ per year. Ambulance costs and added care costs of extra staffing, such as 1:1 for a resident with severe behavioural disturbances are not included in this figure.

Conclusions: Findings suggest that utilizing 2.5 FTE RN/ RPNs with specialized geriatric mental health training and/ or experience distributed over three health service areas have improved the quality of care delivered by residential care front-line staff. They have solidified the use of P.I.E.C.E.S. and GPA as holistic philosophies anchoring mental health/ dementia care and promote the use of the BC BPSD algorithm as the framework for older persons with behavioural and psychological symptoms.

Through the use of coaching, direct and indirect education, nurses, care aides and physicians feel more confident in developing person-centred care strategies and utilizing non-pharmacological interventions first.

Our integrated model, with a focus on collaboration between residential care and Senior's MHSU program, and capacity-building has led to demonstrated cost efficiencies. We are pleased to know that this model will be sustained at the conclusion of the project. We are hoping that a request to increase the number of RKC-CB in the Central Okanagan Health Service Area will be supported given that this area's RKC-CB has twice the number of residential care beds than the other RKC-CBs.

Investing resources into specialized behavioural supports and resources in this way better meets the needs of people with responsive behaviours associated with complex mental health issues, including the dementias and/or other neurological conditions residing in residential care. 